\title{
Environment-Aware Localization of Femtocells for Interference Management
}

\author{
Avishek Patra, Janne Riihijärvi, Jad Nasreddine, Petri Mähönen \\ Institute for Networked Systems, RWTH Aachen University \\ Kackertstrasse 9, D-52072 Aachen, Germany \\ email: $\{$ avp, jar, jad, pma $\} @$ inets.rwth-aachen.de
}

\begin{abstract}
Femtocells are a promising approach to provide high data rates through autonomous configuration in indoor environments. However, due to the random and uncontrolled deployment of femtocells within users premises, interference between femtocells themselves and with macrocell base stations is a major issue. In this work, we look into the interference management problem and work towards the development of an interference mitigation algorithm based on the localization of randomly positioned femtocells using radio environmental information. In particular, we show that based on building floorplans and basic information on the urban landscape, femtocells can accurately localize themselves using macrocellular base stations as anchor nodes. Based on the localized femtocell positions, various channel allocation schemes are employed to mitigate interference.
\end{abstract}

\section{INTRODUCTION}

There has been a fundamental shift in the use of mobile networks from the domination of voice-based communication to that of data communications. According to the survey in [1], the global mobile data usage has been doubling every year since 2006 with the mobile data usage for the year 2011 being 8 -times the total Internet data usage in 2000. To meet the arising requirements for more and more capacity, techniques ranging from reduction of cell size, better modulation schemes and use of smart antenna arrays are developed. However, such techniques are expensive to apply in indoor environments where signal attenuation is high. Even though voice signals can tolerate low signal levels, data signals require high SINR levels to achieve usable bitrates. As a solution, inexpensive, low-power small femtocell base stations have emerged [2]. Femtocell base stations (FBSs) are similar to a macrocell base station (MBS) in terms of major functionality but within a smaller area. The FBSs are user deployed as opposed to being deployed by the operator in a planned fashion as in the MBS case.

To improve capacity and quality of data communications in indoor environments, several heterogeneous networks are being deployed [3]. In such networks, the FBSs cater to a smaller range (radius $=10-50 \mathrm{~m}$ ) while the MBSs serve a larger area (radius $=300-2000 \mathrm{~m}$ ) [4]. FBSs mainly cover the dead zones and indoor environments. In such heterogeneous networks, interference management is a key issue especially due to the uncontrolled way of deploying the FBSs. Although dedicated allocation of spectrum to base stations through heavy frequency reuse prevents inter-tier interference, it is inefficient in terms of spectrum utilization. Compared to dedicated allocation, intra- and inter-tier spectrum sharing is more efficient in spectrum utilization. The disadvantage of spectrum sharing is obviously that it may cause high levels of interference amongst the base stations if not well designed [5]. In literature interference mitigation methods based on frequency hopping, power control and directional antennas have been proposed [6], [7]. In most cases, these methods do not take into account the non-fixed nature of the FBS positions. The FBSs should have self-organization capability in terms of adaptive interference mitigation to cope with the constant relocation of FBSs, placed within users premises.

In this paper we propose an environment-aware localisation based approach for interference management. We show that femtocells can be localized with high accuracy through the use of environmental information such as building floorplans and basic information on the urban landscape, and using as anchor nodes the macrocellular base stations whose locations are known. Our approach is closely related to and can be implemented using the recently proposed Radio Environment Map (REM) approaches for management of future cellular networks [8]. Based on the inferred location information, we focus on location-based co-tier interference management for OFDMA-based FBSs in downlink scenario. The concept can be easily extended to the co-tier uplink and cross-tier scenarios. For the localization based interference mitigation algorithm, FBSs are located using the knowledge of the surrounding environment of the FBSs. To counter the dynamics of the network, FBSs can be periodically located and eventually assigned OFDMA sub-channels.

The rest of the paper is organized as follows. In Section II, we look into key related work. Section III consists of the description of the proposed approach. In Section IV, the results obtained through simulations are shown, demonstrating the performance gains obtained using environmental information. We conclude the paper in Section V summarizing our results and outline future work.

\section{RELATED WORK}

Interference management for femtocells has been a highly active research topic for some time now. Most of the existing work on the topic has focused on mitigating two major categories of interference, commonly defined as follows: 
1) Cross-tier Interference - Occurs between the MBSs and FBSs mainly in the femto-macro boundary region.

2) Co-tier Interference - Takes place between the closely located co-channel FBSs with overlapping channel allocations.

Both cross-tier and co-tier interference mitigation approaches have been studied extensively in the literature, with an overview given in [9]. In this paper our focus will be on the cotier interference, although the approach developed can easily be extended to the cross-tier case as well.

As mentioned in the introduction, our approach is based on FBS localization. For the localization of wireless nodes in indoor scenarios, the global localization systems like GPS are not sufficiently accurate. Signal propagation in indoor environment is unpredictable where reflection and diffraction of signals from multiple walls and indoor objects cause severe multipath interference at the receiver. In most indoor localisation methods proposed [10]-[12], RSSI based methods have been suggested as these do not require special hardware, tight synchronization, or advanced signal processing at the receiver. RSSI-based methods can be easily employed, without additional expenses like directional antennas to achieve high accuracy provided that propagation losses to anchor nodes of known locations are known, or the received signal strengths are mapped beforehand using alternative localization approaches such as manual referencing. The novelty of our approach is the use of radio environmental information to assist in the localization, with the surrounding MBSs acting as anchors for the localization of dynamic FBSs.

Deriving the propagation losses from environmental information requires the use of propagation models. The simplest of these is the log-distance path loss model given as [13]

$$
\operatorname{PL}(d)=\operatorname{PL}\left(d_{0}\right)+10 n \log \left(\frac{d}{d_{0}}\right),
$$

where PL is path loss and $n$ is the path loss exponent. The coefficients for the relation are determined by obtaining a curve-fit for the variation between distance and path loss. Better results can be obtained using semi-empirical models [14] and more advanced parametric models such as ray-tracing models [15]. For the propagation of radio signals from outdoor to indoor scenarios, the radio waves are highly influenced by the outer walls, wall materials and the internal organization of the building. The loss in signal strength depends on the reflection and transmission coefficients of walls. There are various outdoor-to-indoor propagation models which are a combination of outdoor and indoor models with special consideration regarding the transmission of radio wave from outdoor to indoor environment. While in COST 231 Model, the radio waves penetrate through the outer walls to enter a building, in the work in [16], the entry points are assumed to be through openings like doors and windows. Unfortunately, real path losses cannot be accurately represented by deterministic path loss models. Therefore, we use in this paper a method based on maximum likelihood to remove the uncertainty.

\section{ENVIRONMENT-AWARE INTERFERENCE MANAGEMENT FOR FEMTOCELLS}

Our proposed Environment-Aware Interference Management (EIM) algorithm can be divided into two separate parts, namely an algorithm for localization of individual femtocells using environmental information, and a family of interference management approaches based on the localization information. We shall explain both of these in detail in the following.

\section{A. Localization of Femtocells}

For the localization process, environmental models of the indoor and the urban environment are used. Indoor model is required for the building containing the FBS whereas the urban model replicates the surrounding environment of the building. Urban models contain information such as the locations and heights of the buildings, commonly available through the planning tools used by the network operators. Indoor models give a 3D description of the indoor environment and contain information such locations and types of the walls. Such information can be made available from the plans of the building through tools that can extract position of walls from floor plans. Based on the urban and indoor environmental information, the localization algorithm consists of two parts: database generation and localization based on database referral.

1) Database Generation: The databases generated (further known as indoor Calculated-Power Databases (CPDBs)), contain $x$-coordinates, $y$-coordinates and the Calculated Received Power (CRP) at different equidistant points through the indoor environment. CPDBs are calculated with respect to each MBS. These are illustrated in Figure 1. The measurement points $P_{i}$ are separated by a specific distance depending on the required resolution. The CRP at a point $P_{i}$ depends on the transmitted power, path loss due to urban environment and penetration loss due to indoor environment. While path loss is calculated based on the distance between MBS and $P_{i}$, urban structural model and outdoor propagation model, the indoor structural data and indoor propagation models are used to obtain penetration loss. The path loss can be given as

$$
\lambda_{\text {path }}=F\left(d, n_{\text {buildings }}, h_{\text {buildings }}\right),
$$

where, $\lambda_{\text {path }}$ is the urban path loss, $d$ is the distance between the given MBS and $P_{i}, n_{\text {buildings }}$ is the number of buildings between the given MBS and $P_{i}$ and $h_{\text {buildings }}$ is the average height of buildings between the given MBS and $P_{i}$. The path loss function $F$ can be selected to be of a simple parametric form, such as the extended Walfish-Ikegami model [18]. Similarly, penetration loss can be depicted as

$$
\lambda_{\text {pen }}=n_{\text {walls }} \Psi,
$$

where $\lambda_{\text {pen }}$ is the penetration loss, $\Psi$ is the transmission and reflection loss (which depends on the thickness and material of walls, frequency of operation and angle of incidence of the signal), and $n_{\text {walls }}$ is the number of walls between the points $P_{i}$ within the considered building and the given MBS. 

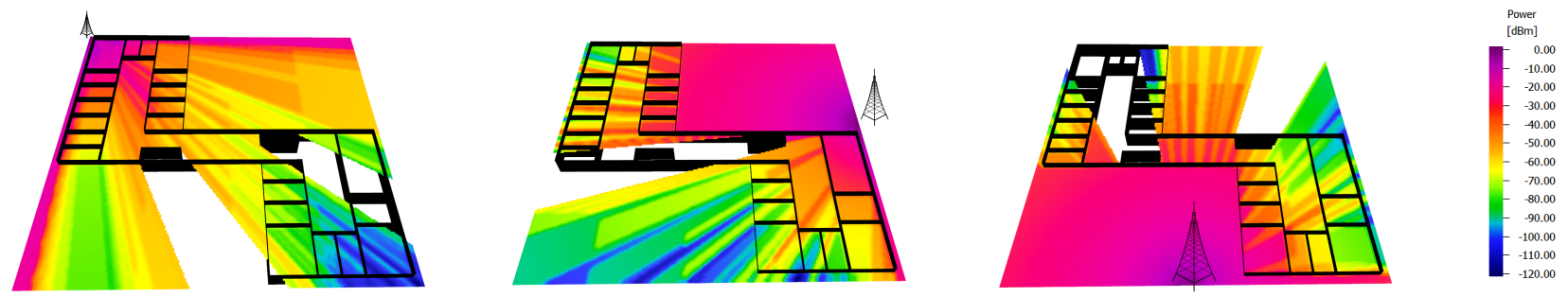

Fig. 1. Illustration of large differences in received signal strengths from three nearby base stations (blue dots) induced by the indoor environment. For purposes of illustration of the differences in arising signal strengths the base stations have been placed very close to the building (akin to picocellular base stations). For the simulations carried out in the paper realistic base station distances are used instead.

Thus, CRP at $P_{i}$ is the difference of the transmission power of the given MBS and the total loss in signal strength given by $\lambda_{\text {pen }}+\lambda_{\text {path }}$. The results, namely CRP and the $x$ - and the $y$-coordinates of $P_{i}$ are stored in the indoor CPDB for the given MBS. CPDBs are obtained for all the MBSs.

We emphasize that the CRP values can either be obtained through sufficiently advanced propagation simulations, or through measurement based approach where the propagation models are calibrated through measurements conducted by mobile terminals and femtocells. The latter can significantly increase the accuracy of the approach in the presence of uncertainties in the indoor propagation characteristics.

2) Localization based on Database Referral: The CPDBs for a FBS-cluster are made available to the Femto-Gateway through, for example, local Radio Environment Map (REM) instance. When an FBS is to be localized, the REM refers to the CPDBs. Each FBS receives real-time signals from the reference MBSs, referred to as the Actual Received Powers (ARPs). For a given MBS $m$, the FBS receives $\mathrm{ARP}^{m}$ from MBS $m$ ( $m \in\{1, \ldots, M\}$ where $M$ is the number of MBSs). The comparison of the CRPs from indoor CPDBs and the ARPs, for the respective MBSs helps the FBS to localise itself. If the ARPs from different MBSs at a point match the CRPs of the MBSs at that point, the FBS is predicted to be located at that point. However, finding an exact power match between $\mathrm{ARP}^{m}$ and $\mathrm{CRP}^{m}$ values of indoor CPDBm is highly improbable as the ARPs are shadowed and faded compared to the simple path loss estimates. Hence, to obtain the location of the FBS, the unwanted shadowing effect on the ARPs must be taken into account.

For each MBS, the shadowing probability density function (PDF) is obtained for the respective MBSs from the urban propagation model. Instead of getting an exact location, we aim to obtain the maximum likelihood location of the FBS using the shadow PDFs. When ARPs are received by a FBS, for a given MBS $m$, the effective shadow loss is obtained by subtracting the $\mathrm{CRP}^{m}$ values of all points $P_{i}$ in the building (obtained from $\mathrm{CPDB}^{m}$ ) and $\mathrm{ARP}^{m}$. Based on the shadow PDF of the MBS $m$, a likelihood probability is associated to each point $P_{i}$. Applying this procedure for all the $m$ MBSs, each point gets $m$ likelihoods assigned to it. The overall likelihood for each point $P_{i}$ is obtained from the product of individual likelihood probabilities. The point with the maximum overall likelihood is the maximum likelihood solution for the localization problem. We also define the maximum likelihood room, which is the room where the maximum likelihood point is. The latter is useful for interference management problems, as the interference relations between femtocells and also across tiers are often dominated by the wall losses, instead of precise positions of the nodes within the rooms.

\section{B. Channel Allocation Schemes}

To manage interference between the FBSs in the indoor environment, OFDMA sub-channels are allocated to the FBSs based on the determined locations. Channel allocation based on location knowledge ensure that all the User Equipments (UEs) catered by the FBSs have higher SINR compared to when allocation is done without location information. As the optimal allocation of channels is NP-hard, different heuristic and meta-heuristic methods are commonly used for channel allocation. These methods are able to approximate the optimal condition with little error. In this work, the frequency assignment is done using two different methods described in detail in the following.

1) Graph Colouring Method: Here the channel allocation problem is mapped as a graph coloring problem, where the different FBSs are treated as the vertices $V$ of an interference graph $G=(V, E)$ and interference between the FBSs is represented through edges $E$. The available sub-channels are color coded, and these colors are marked to the vertices of the interference graph with the condition that any two neighboring vertices do not have the same color. This ensures there is no co-channel interference. As graph coloring is an NP-Hard problem, the allocation time increases drastically with the increase of graph size. Hence, to get a solution within practical limits, certain heuristic methods like DSATUR algorithm [17] have been proposed in literature. The DSATUR algorithm gives a very good approximation of the actual solution, and we adopt it as one foundation for our interference management scheme.

2) Simulated Annealing Method: Simulated annealing [19], [20] is a probabilistic meta-heuristic method for solving global optimization problems that gives good results even in presence of large search spaces. SAM has served well in allocation of channels in 802.11 WLAN Networks [21] and for UMTS [22]. In this work, to obtain a channel allocation with the least cochannel interference, the objective function, $\Psi$ is defined as 
the sum of interference at all the range points of all the FBSs due to a given channel allocation. The steps of the simulated annealing algorithm are then as follows:

(a) Initially, randomly allocate channel and set the initial temperature, $T=T_{0}$. Calculate the initial objective function value, $\Psi_{0}$ for the initial channel allocation, $C=C_{0}$.

(b) At the end of the $n$th iteration $\left(T=T_{n} ; \Psi=\Psi_{n}\right)$, the current channel allocation $C_{n}$ is obtained.

(c) For the $(n+1)$ th iteration, new channel allocation, $C_{n+1}$ is obtained as one or more FBSs randomly change their previously allocated channels. Based on $C_{n+1}, \Psi_{n+1}$ and $T_{n+1}$ are calculated.

(d) If there is a decrease in $\Psi_{n+1}$ (i.e. $\Psi_{n}-\Psi_{n+1}$ or $\Delta \Psi \leq$ 0 ), then the current allocation is replaced by the new allocation $\left(C=C_{n+1}\right)$.

(e) If there is an increase in $\Psi_{n+1}$ (i.e. $\Delta \Psi \geq 0$ ), then the new allocation is accepted with probability $P$, where $P$ is given by $P=\exp \left(\Delta \Psi / T_{n+1}\right)$. If the new allocation is accepted then the current allocation is replaced by the new allocation $\left(C=C_{n+1}\right)$. Else, the current allocation remains the same $\left(C=C_{n}\right)$.

(f) Steps (b)-(e) continue with the temperature $T$ decreased at each time step until $T=0$. The current allocation at the end of last iteration is the final allocation.

\section{PERformance Evaluation}

The localization algorithm and the different channel allocation schemes are tested in different indoor environments for different propagation models and reference MBS deployment scenarios. Depending on the channel allocation, the average downlink SINR values for the different scenarios are calculated. These SINR values are used to analyze the variations of downlink SINR values with different FBS deployment scenarios.

\section{A. Localisation Performance}

The implementation and verification of the localization algorithm is carried out in a realistic simulation of an urban environment of a large city performed using the WinProp propagation simulator. Detailed indoor models of single and double storied multi-room buildings were obtained to study the indoor environment. The heights of the buildings are $5 \mathrm{~m}$ and $10 \mathrm{~m}$, respectively, with varying room sizes and typical wall thickness of $10 \mathrm{~cm}$. The frequency of transmission of MBSs and FBSs is $2000 \mathrm{MHz}$. The localization process depends on the deployment and transmission characteristics of the reference MBSs. In this work, MBSs are assumed to have a transmission power of $10 \mathrm{~W}$ and transmit using omnidirectional antennas. The transmitters are located at a height of $15 \mathrm{~m}$ from ground level. The received power values are measured at height of $1.5 \mathrm{~m}$ for single-storied building and at heights of $1.5 \mathrm{~m}$ and $6.5 \mathrm{~m}$ from ground level for doublestoried building respectively. Different simulation scenarios considered are shown in Table I.

From the simulations, the location of the FBSs can be correctly predicted within a room with an accuracy of $88 \%$ in
TABLE I

SIMULATION SCENARIOS FOR FEMTOCELL LOCALISATION.

\begin{tabular}{lll}
\hline Scenario & MBS count & Propagation model \\
\hline A & $8 \mathrm{MBSs}$ & COST 231 Walfisch-Ikegami \\
B & $8 \mathrm{MBSs}$ & Measurement-based fit to extended Walfisch-Ikegami \\
C & $6 \mathrm{MBSs}$ & COST 231 Walfisch-Ikegami \\
D & $6 \mathrm{MBSs}$ & Measurement-based fit to extended Walfisch-Ikegami \\
\hline
\end{tabular}

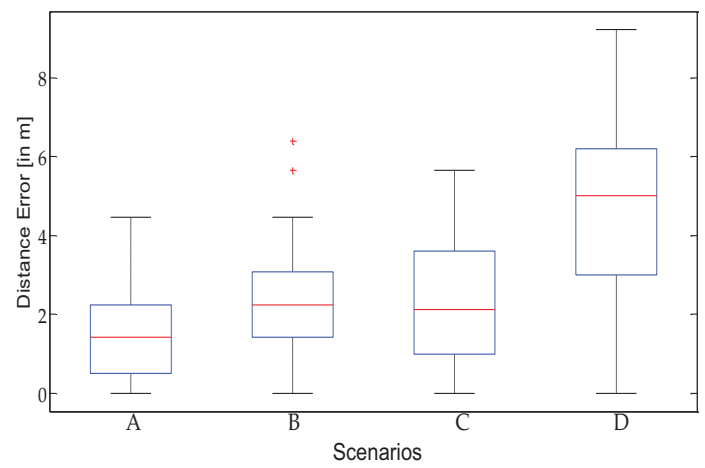

Fig. 2. Summary of localization performance using environmental information. The three lines of the boxes correspond to median, 25th and 75th percentile of localization error, with the whiskers extending to data points at most 1.5 times the interquartile range from the edge of the box.

scenario $\mathrm{A}$ and $64 \%$ in scenario B. For scenario $\mathrm{C}$ and $\mathrm{D}$, the accuracy is less, being $54 \%$ and $10 \%$ respectively. Figure 2 summarizes the localization performance for the considered scenarios. The distance error, i.e. the distance between the actual location and the predicted position of the FBS was also determined. The mean distance error in scenario A is $1.22 \mathrm{~m}$ while in $\mathrm{D}$, it is $4.43 \mathrm{~m}$. Distance error is zero for about $30 \%$ of simulation runs in scenario A and about $15 \%$ of simulation runs in scenario B. As can be seen in the results of scenarios A-D, there is a variation in the accuracy in localization of FBS due to different propagation model for generating CPDB. Also, for different MBS deployment scenarios, given that the propagation models for indoor CPDBs are the same (as in scenarios $\mathrm{A} / \mathrm{C}$ and $\mathrm{B} / \mathrm{D}$ ), there can be considerable difference in the performance of the algorithm. The variability of distances between the MBSs and the FBS also plays a role in the results, as higher variability means higher contrasts in received powers, leading to better predictions. Overall, for dense urban environments with several nearby MBSs, very good localization performance is achieved by exploiting environmental information.

\section{B. Interference Management Performance}

The two selected interference management schemes are simulated under different FBS deployment scenarios and indoor environments assuming that the FBSs have been located with the accuracy as determined in the previous section. The simulation scenarios are summarized in Table II. Depending on the channel allocation, the average FBS SINR is calculated by averaging all the FBS range points within (a) Building, 
TABLE II

SIMULATION SCENARIOS FOR INTERFERENCE MANAGEMENT SCHEMES.

\begin{tabular}{lllll}
\hline & \multicolumn{2}{c}{ FBS deployment } & & \\
\cline { 2 - 3 } Scenario & Number of femtos & Number of channels & Indoor environment & Interference management scheme \\
\hline A1 & 8 & 3 & Single-storied apartment building & Graph coloring \\
A2 & 8 & 3 & Single-storied apartment building & Simulated annealing \\
B1 & 12 & 4 & Single-storied apartment building & Graph coloring \\
B2 & 12 & 4 & Single-storied apartment building & Simulated annealing \\
C1 & 20 & 6 & Two-storied apartment building & Simulated annealing \\
C2 & 20 & 8 & Two-storied apartment building & Simulated annealing \\
D1 & 30 & 6 & Two-storied apartment building & Simulated annealing \\
D2 & 30 & 8 & Two-storied apartment building & Simulated annealing \\
\hline
\end{tabular}

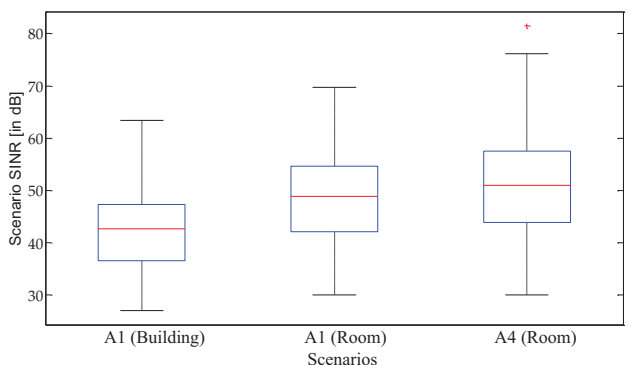

(a)

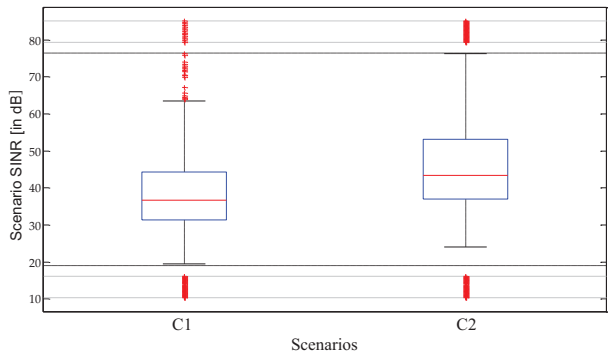

(c)

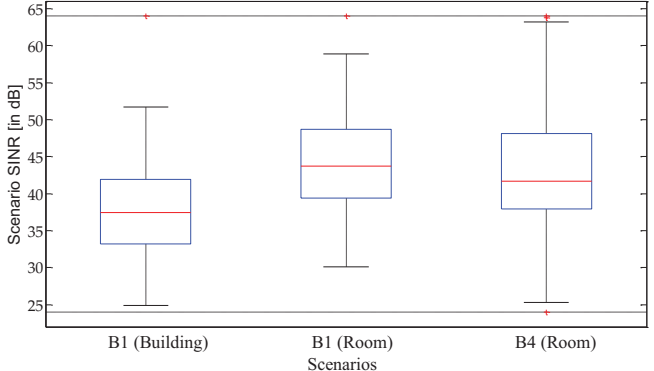

(b)

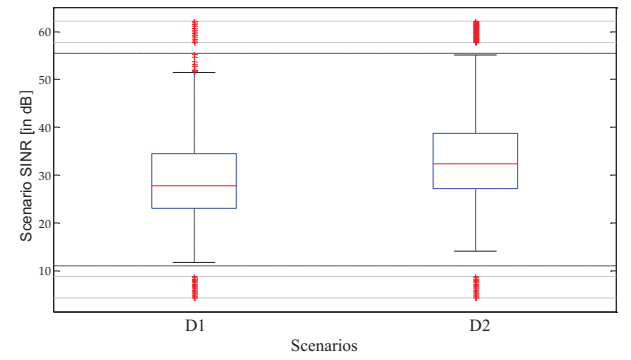

(d)

Fig. 3. Performance of interference mitigation techniques: (a) Boxplots for Scenarios A1 (Building \& Room) \& A4 (Room), (b) Boxplots for Scenarios B1 (Building \& Room) \& B4 (Room), (c) Boxplots for Scenarios C1 \& C2, and (d) Boxplots for Scenarios D1 \& D2 with MDS $=-40 \mathrm{dBm}$.

and (b) Room containing the FBS. The Average FBS SINR is calculated as the mean ratio of the received signal strength to the co-channel interference power and noise power. Noise of course depends on the bandwidth of each channel. In this work, the overall bandwidth is $10 \mathrm{MHz}$ and each sub-channel has bandwidth of $(10 / \eta) \mathrm{MHz}$ ( $\eta$ is the number of channels), and the noise power is assumed to be given by the corresponding thermal noise. Note that the simulations are done by randomly placing the FBSs across the indoor environment for multiple simulation runs. The average scenario SINR is calculated as the average of the FBS average SINRs over all the simulation realizations. For the simulations, Minimum Detectable Signal (MDS) strength of -40 and $-50 \mathrm{dBm}$ is used for interference graph generation for graph coloring approach. The average scenario SINRs calculated for the different scenarios are shown in Tables III and IV. Figures 3 (a)-(d) show the comparison of the statistical analysis of the average scenario SINRs for the different scenarios for MDS
TABLE III

SINR DISTRIBUTION FOR ALL SCENARIOS (MDS $=-40$ DBM).

\begin{tabular}{lllll}
\hline Scenario & Method & Mean & 5th Percentile & 95th Percentile \\
\hline A1 & Building & 43.02 & 31.51 & 59.14 \\
& Room & 49.41 & 34.31 & 67.85 \\
A4 & Room & 51.31 & 35.07 & 68.52 \\
B1 & Building & 38.65 & 27.05 & 48.22 \\
& Room & 44.47 & 30.47 & 56.66 \\
B4 & Room & 43.27 & 28.98 & 59.26 \\
C1 & Room & 40.04 & 26.23 & 62.01 \\
C2 & Room & 49.43 & 31.29 & 111.4 \\
D1 & Room & 30.11 & 17.16 & 47.49 \\
D2 & Room & 35.40 & 21.91 & 60.93 \\
\hline
\end{tabular}

of $-40 \mathrm{dBm}$ and $-50 \mathrm{dBm}$.

For the previous simulations, channel allocations were done for accurately located FBSs. However, as we saw earlier, 
TABLE IV

SINR DISTRIBUTION FOR SCENARIOS A1 AND B 1 (MDS $=-50$ DBM).

\begin{tabular}{lllll}
\hline Scenario & Method & Mean & 5th Percentile & 95th Percentile \\
\hline A1 & Building & 34.30 & 22.83 & 51.09 \\
& Room & 48.72 & 33.47 & 67.22 \\
B1 & Building & 31.31 & 22.12 & 40.87 \\
& Room & 45.77 & 36.12 & 58.17 \\
\hline
\end{tabular}

TABLE V

IMPACT OF LOCALIZATION INACCURACY ON AVERAGE SCENARIO SINR WITHIN A BUILDING.

\begin{tabular}{lllll}
\hline Method & Localisation & Mean & 5th Percentile & 95th Percentile \\
\hline \multirow{2}{*}{ Building } & Accurate & 41.79 & 26.22 & 107.18 \\
& Inaccurate & 19.60 & 1.72 & 103.41 \\
\multirow{2}{*}{ Room } & Accurate & 48.77 & 31.30 & 111.51 \\
& Inaccurate & 22.35 & 3.38 & 101.10 \\
\hline
\end{tabular}

accurate localization is not possible in all of the simulation scenarios. Further simulations were done to compare the average scenario SINRs for allocation in case of inaccurate localization and accurate localization. In these simulations, presented here only for Scenario B for space reasons, the locations of FBSs within the circle with radius of 95th percentile of the distance error $(4.47 \mathrm{~m})$ are randomly selected. Comparison of scenario SINR is done for scenario A1 (MDS $=-40 \mathrm{dBm})$. Thus, depending on the changed position but same channel allocation, room and building based Average FBS SINRs are calculated for all the FBSs. It should be noted that the random positions of FBS within this error circle are not limited within the maximum likelihood room of FBS. Hence, two cases are taken into consideration. In Case 1, the random positions within the building are chosen whereas in Case 2, only the random positions within the maximum likelihood room containing FBS are chosen. Table V show the variation in average scenario SINR for scenario A1 in case of accurate and inaccurate localization. It can be observed that the mean SINR variation in case 1 is $22.19 \mathrm{~dB}$ when range points within building are considered, while it is $26.42 \mathrm{~dB}$ when range points within room is considered. Note that the very high SINR values for the 95th percentile occur due to very close by placements of the FBS and mobile terminal for certain FBS locations, and due to the simulations not considering the near field effects of the transceivers.

\section{CONCLUSiOnS}

In this paper we have shown how environmental information can be used for accurate femtocell localization and interference management. Our proposed algorithm can locate femtocell base stations within a room with a maximum probability of $88 \%$ with the average error in localization between $1.36 \mathrm{~m}$ and $4.65 \mathrm{~m}$ for the empirical and parametric propagation models. Channel allocation using heuristic and meta-heuristic methods like simulated annealing and graph coloring yield minimum average SINR of $19.61 \mathrm{~dB}$ and a maximum average SINR of
$51.31 \mathrm{~dB}$ for the downlink users for the simulated scenarios. The results show that the Environment-aware Interference Management (EIM) algorithm for FBSs proposed in this work can be highly effective in localizing FBSs in indoor environments and in mitigation of co-channel interference.

\section{ACKNOWLEDGEMENT}

We thank RWTH Aachen University and the German Research Foundation (Deutsche Forschungsgemeinschaft, DFG) for providing financial support through the UMIC research centre.

\section{REFERENCES}

[1] Cisco white paper, "Cisco visual networking index: Global mobile data traffic forecast update, 20102015," Feb. 2011.

[2] H. Claussen, L. T. W. Ho, L. G. Samuel, "An overview of the Femtocell concept,” Bell Labs Tech. Journal, vol. 13, no. 1, pp.221-245, May 2008.

[3] J. Shapira, "Microcell engineering in CDMA cellular networks," IEEE Tran. on Vehicular Technology, vol. 43, no. 4, pp. 817-825, Nov. 1994.

[4] A. Ganz, C. M. Krishna, D. Tang, Z. J. Haas, "On optimal design of multitier wireless cellular systems," IEEE Communications Magazine, vol. 35, no. 2, pp. 88-93, Feb. 1997.

[5] V. Chandrasekhar, et al., "Power control in two-tier Femtocell networks," IEEE Trans. on Wireless Comm., 2008.

[6] V. Chandrasekhar, J. G. Andrews, "Spectrum Allocation in Tiered Cellular Networks", IEEE Transactions on Communications, Vol. 57, No. 10, pp. 3059-3068, October 2009.

[7] H. Claussen, "Performance of Macro- and Co-Channel Femtocells in a Hierarchical Cell Structure," in Proc. of IEEE PIMRC 2007, pp.1-5, 3-7 Sept. 2007.

[8] T. Cai, et al., "Design of layered radio environment maps for ran optimization in heterogeneous LTE systems," in Proc. of IEEE PIMRC 2011, September 2011, pp. $172-176$.

[9] N. Saquib, E. Hossain, L.B. Le, D.I. Kim, "Interference management in OFDMA Femtocell networks: issues and approaches", IEEE Wireless Commun. 2012, pp.86-95.

[10] Y. Ohta, M. Sugano, M. Murata, "Autonomous localization method in wireless sensor networks," in Proc. of IEEE PerCom 2005 Workshops, pp. 379-384, 8-12 March 2005.

[11] M. Sugano, T. Kawazoe, Y. Ohta, M. Murata, "Indoor Localization System using RSSI Measurement of Wireless Sensor Network based on ZigBee Standard", Wireless and Optical Communications, 2006.

[12] N.A. Dieng, C. Chaudet, M. Charbit, L. Toutain, T. Ben Meriem, "Experiments on the RSSI as a Range Estimator for Indoor Localization," in Proc. of NTMS 2012, pp.1-5, 7-10 May 2012.

[13] T. S. Rappaport, "Wireless Communications: Principles and Practice", 2nd edition.

[14] M. Döhler, "An Outdoor-Indoor Interface Model for Radio Wave Propagation for 2.4, 5.2 and $60 \mathrm{GHz}$ ", Master Thesis Report, 1998-99.

[15] T.K. Sarkar, et al., "A survey of various propagation models for mobile communication," Antennas and Propagation Magazine, IEEE , vol.45, no.3, pp. 51-82, June 2003.

[16] Y. Miura, Y. Oda, T. Taga, "Outdoor-to-Indoor Propagation Modelling with the Identification of Path Passing Through Wall Openings", in Proc. of IEEE PIMRC 2002, vol.1, pp.130-134.

[17] D. Brlaz, "New Methods to Color the Vertices of a Graph," Comm. ACM 22, 251-256, 1979.

[18] T. Schwengler, M. Glbrt, "Propagation models at $5.8 \mathrm{GHz}-$ path loss and building penetration' ', in Proc. of IEEE RAWCON 2000, pp. 119-124.

[19] S. Kirkpatrick, C. Gelatt, Jr., M. Vecchi, "Optimization by simulated annealing," Science, Vol220, No 4598, May 1983, pp. 671-680.

[20] V. Cerny, "Thermodynamical approach to the travelling salesman problem: An efficient simulation algorithm", J. Optim. Theory Appl. 45:41$51,1985$.

[21] J. Chen, S. Olafsson, X. Gu, "Observations on Using Simulated Annealing for Dynamic Channel Allocation in 802.11 WLANs," in Proc. of IEEE VTC Spring 2008, pp. 1801-1805.

[22] J. Nasreddine, X. Lagrange, "On The Computation of the Maximum Capacity of TDMA-CDMA/TDD Systems," in Proc. of IEEE PIMRC 2006, Helsinki, Finland, September 2006. 